\title{
Development and Application of MRF Based on Robot Arm
}

\author{
Zhang Xuejun $^{1,2} *$, Li Longxiang ${ }^{1,2 * *}$, Xue Donglin ${ }^{1,2}$, Song Chi ${ }^{1,2}$, , Ai Bo ${ }^{1,2}$ \\ ${ }^{1}$ Key Laboratory of Optical System Advanced Manufacturing Technology, Chinese Academy of Sciences, Changchun 130033, China \\ ${ }^{2}$ Changchun Institute of Optics, Fine Mechanics and Physics, Chinese Academy of Sciences, Changchun 130033, China
}

\begin{abstract}
Magnetorheological Finishing (MRF) is widely regarded as an effective technique to polish and figure aspheric optics. MRF based on Robot Arm is developed by us. This new machine is more flexible, efficient, cost-effective and smaller space-usage in optical shop, compared with the traditional MRF machines. The components of MRF based on Robot Arm are introduced firstly. Position-attitude control and polishing tool path are also studied in this paper. The experiments and application of MRF based on Robot Arm demonstrate the effectiveness and validity of MRF based on Robot Arm in optical fabrication.
\end{abstract}

\section{Introduction}

Magnetorheological Finishing (MRF) is a typical, deterministic sub-aperture computer-controlled opticalsurfacing technology [1] and widely regarded as an effective means of processing aspheric optical components due to its high deterministic tool influence function(TIF) and excellent surface roughness quality[25]. MRF is originally invented by Dr. William Kordonski and his colleagues in Belarus and commercialized by QED Technologies in USA [6]. In those commercialized MRF machines, such as Q22-950 and Q22-1200[7], a module of MRF is integrated into traditional machine which is called 'Box-machine' of MRF in this paper. The Box-machine of MRF is characterized by high position-accuracy and high workstability. They has proved the capability of MRF by high-precision and fast polishing aspherical optics. However, such Box-machine has some weakness in optical shop. For example, Box-machines has an expensive price and high equipment cost. A large space is necessary for Box-machines. Moreover, the machine motion performance including velocity and acceleration, is limited when the size of Box-machine become large.

In order to solve these problems mentioned above in optical shop, we developed a new type of MRF machine based on Robot Arm. A specially designed MRF module was integrated into the robot arm who adopts a 6-axis series structure. This type of MRF machine has high motion performance and six degrees of freedom, which is flexible enough to manufacture aspherical or free-from optics. It also has a smaller space occupation and lower equipment cost compared with Box-machine. These advantages make the MRF based on Robot Arm a high cost-effective polishing equipment.

\section{Components of MRF based on Robot Arm}

The working wheel of the MRF module is $\$ 360 \mathrm{~mm}$ and $\Phi 160 \mathrm{~mm}$ with a permanent magnet[8]. The MRF module is integrated into the Robot Arm as shown in Fig. 1(a) and (b). The layout of Magnetorheological Fluid cycle system was carried out reasonably to ensure the stability of the whole MRF system on Robot Arm.

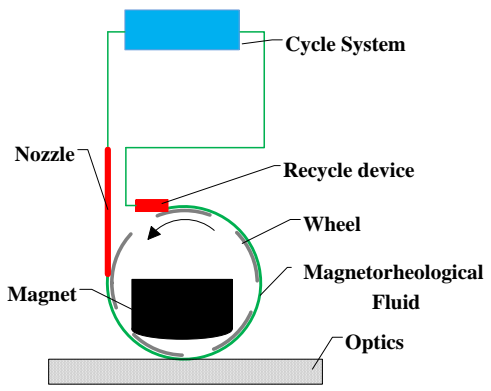

(a) Details of components

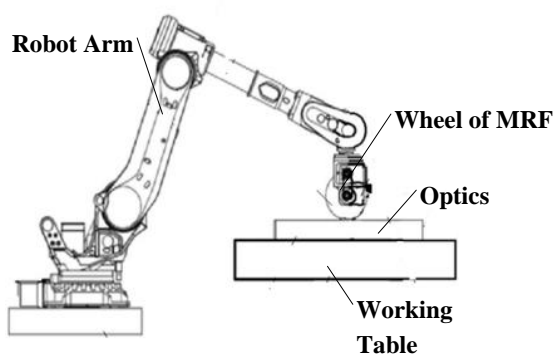

(b) The removal function of MRF

Fig. 1 The MRF based on Robot Arm

\section{Position-attitude control of MRF based on Robot Arm}

One of the position-attitude control principles for the MRF process requires that the normal direction of the outer surface of the MRF working wheel is aligned with the normal direction of the local aspherical surface[9]. Fig. explains the ZYX Euler angle of the Robot Arm. The definition of rotation transformation is shown in Equation (1).

*Corresponding author: zxj@ ciomp.ac.cn; **lilx@ ciomp.ac.cn 

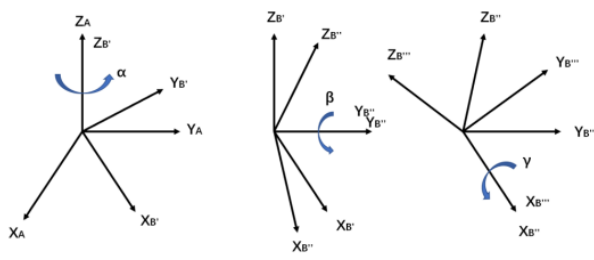

Fig. 2 ZYX Euler angle

$$
\begin{gathered}
{ }_{B}^{A} R_{Z^{\prime} Y^{\prime} X^{\prime}}=R_{Z}(\alpha) R_{Y}(\beta) R_{X}(\gamma)= \\
{\left[\begin{array}{ccc}
c \alpha & -s \alpha & 0 \\
s \alpha & c \alpha & 0 \\
0 & 0 & 1
\end{array}\right]\left[\begin{array}{ccc}
c \beta & 0 & s \beta \\
0 & 1 & 0 \\
-s \beta & 0 & c \beta
\end{array}\right]\left[\begin{array}{ccc}
1 & 0 & 0 \\
0 & c \gamma & -s \gamma \\
0 & s \gamma & c \gamma
\end{array}\right]}
\end{gathered}
$$

This method of transforming angle of MRF based on Robot Arm, is meaningful in theoretical calculation and initial simulation. But sometimes it is not enough to use the system to conduct practical optical processing. It is often needed to introduce quaternion or some parameter restrictions on the rotation direction of a specific axis to achieve the unique solution of the robot, so as to achieve the purpose of making the actual trajectory of the system consistent with our requirements. The transformation equation from Euler angle to quaternion is shown in Equation

$$
\left[\begin{array}{l}
Q 1 \\
Q 2 \\
Q 3 \\
Q 4
\end{array}\right]=\left[\begin{array}{l}
c\left(\frac{\alpha}{2}\right) c\left(\frac{\beta}{2}\right) c\left(\frac{\gamma}{2}\right)+s\left(\frac{\alpha}{2}\right) s\left(\frac{\beta}{2}\right) s\left(\frac{\gamma}{2}\right) \\
s\left(\frac{\alpha}{2}\right) c\left(\frac{\beta}{2}\right) c\left(\frac{\gamma}{2}\right)-c\left(\frac{\alpha}{2}\right) s\left(\frac{\beta}{2}\right) s\left(\frac{\gamma}{2}\right) \\
c\left(\frac{\alpha}{2}\right) s\left(\frac{\beta}{2}\right) c\left(\frac{\gamma}{2}\right)+s\left(\frac{\alpha}{2}\right) c\left(\frac{\beta}{2}\right) s\left(\frac{\gamma}{2}\right) \\
c\left(\frac{\alpha}{2}\right) c\left(\frac{\beta}{2}\right) s\left(\frac{\gamma}{2}\right)-s\left(\frac{\alpha}{2}\right) s\left(\frac{\beta}{2}\right) c\left(\frac{\gamma}{2}\right)
\end{array}\right]
$$

\section{Application of MRF based on Robot Arm}

A $1000 \mathrm{~mm} \times 470 \mathrm{~mm}$ off-axis aspherical mirror is also processed by MRF based on Robot Arm. The material of the mirror is also $\mathrm{SiC}$ with a layer of $\mathrm{Si}$ on the surface. The initial surface accuracy is $45.76 \mathrm{~nm}$ rms as shown in Fig. (a). Considering the risk for this project-used mirror, a high-precision residual surface error is not pursued within only one polish-test iteration, therefore the total time of one iteration is set to 7 hours. After 7 hours effectively polishing as shown in Fig. (b), the rms of the surface error map of the mirror is converged to $22.78 \mathrm{~nm}$ as shown in Fig. (c). Although the convergence rate is about $50.2 \%$, the determinacy of MRF based Robot Arm is also very high considering the limit of total polishing time in one cycle. This proves the MRF based Robot Arm is also effective and valid for aspherical optics.
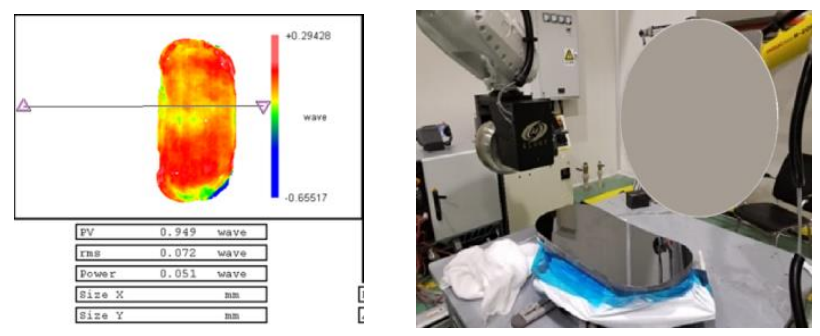

(a) I nitial surface error map (b) Actual process by MRF

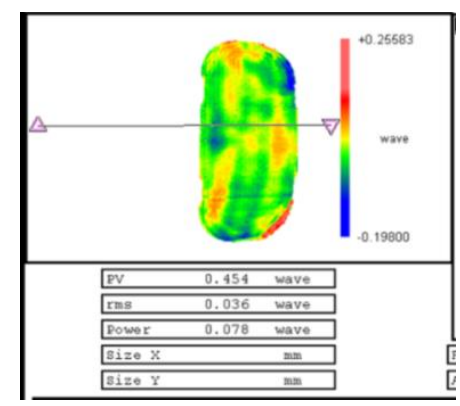

(c) The residual surface error map

Fig. 3 Off-axis aspherical mirror is processed 7 hours

\section{Conclusion}

Developments and application of MRF based on Robot Arm are introduced in this paper. The application in real optics fabrication proves that this new MRF based on Robot Arm is effective and valid for high-precision plane and curved optical surface. Compared to traditional MRF machine, the MRF based on Robot Arm developed by us is more flexible, efficient, cost-effective and smaller space-usage in optical shop. More work about MRF and robot arm will be done in the future.

\section{References}

[1] Li L, Xue D, Deng W, et al. Positive dwell time algorithm with minimum equal extra material removal in deterministic optical surfacing technology.[J]. Applied Optics, 2017, 56(32):9098.

[2] Kordonski W I, Jacobs S D . Magnetorheological Finishing [J]. International Journal of Modern Physics B, 1996, 10(23n24):2837-2848.

[3] Golini D, Kordonski W I, Dumas P. Magnetorheological finishing (MRF) in commercial precision optics manufacturing[J]. Proceedings of SPIE The International Society for Optical Engineering, 1999, 3782:80-91.

[4] Golini D, Kordonski W I, Dumas P. Precision optics fabrication using magnetorheological finishing[J]. Critical Reviews, 2017, 67:251-274.

[5] Yang C, Peng X, Hu H, et al. Stability Control of Magnetorheological Finishing Removal Function Under Continuous Process Status[J]. Nanotechnology \& Precision Engineering, 2016.

[6] Harris, Daniel C. History of Magnetorheological Finishing [J]. Proceedings of SPIE - The International Society for Optical Engineering, 2011, 8016(4):561-566. [7] https://qedmrf.com/en/mrfpolishing/mrfproducts/q22-950

[8] Li L, Zheng L, Deng W, et al. Optimized dwell time algorithm in magnetorheological finishing[J]. International Journal of Advanced Manufacturing Technology, 2015, 81(5-8):833-841. 